\title{
LA RICEZIONE DEI RACCONTI DEL CALILA E DIMNA IN ITALIA
}

\author{
Gaetano LALOMIA \\ Università degli Studi di Catania \\ glalomia@unict.it
}

La narrativa breve medievale è per sua natura portata a nutrirsi di svariate forme codificate; è un materiale assorbente che riesce a catturare storie da qualsiasi contenitore, per poi operare una profonda rielaborazione dei temi secondo le finalità con cui la voce narrante, scritta od orale che sia, intende raccontare la storia. Tracciare una sorta di mappa dei racconti che viaggiano nel Medioevo romanzo non è facile, ma diventa in un certo senso necessario per poter stabilire non solo quali sono i testi e i racconti che danno vita alla narrativa breve romanza in Occidente, ma anche quali attecchiscono nel tempo, divenendo, agli occhi dei fruitori, delle narrazioni proprie, e così proprie da dimenticare la loro origine.

È con questo scopo che, accogliendo l'invito di María Jesús Lacarra, a partecipare alla vita di questo volume dedicato al racconto medievale, intendo operare una verifica della ricezione del Calila e Dimna nell'Occidente romanzo, con particolare attenzione all'Italia. Non si tratta di analizzare la ricezione dell'opera nella sua interezza, quanto i singoli racconti nella loro rielaborazione da parte delle culture riceventi. Siffatto rilevamento permette altresì di radiografare, accanto alla ricezione dell'opera nella sua interezza, la misura del viaggio dei singoli testi. Si tratta, in altre parole, di aggiungere un tassello in più a un dato ormai evidente a tutti, cioè che la cultura occidentale si è andata formando, nel Medioevo, sulla base di diverse tradizioni; si tratta, inoltre, di tentare di ricostruire, per grandi linee generali (dove è possibile farlo), i tasselli che hanno dato vita alle forme letterarie della modernità. 


\section{L'arrivo del Calila e Dimna in Occidente}

L'arrivo del Calila e Dimna in Occidente è legato inevitabilmente alle considerazioni più generali che investono gli influssi culturali orientali sulla cultura occidentale. Grazie agli studi intrapresi a partire dalla fine degli anni '70 del Novecento, che hanno valorizzato la narrativa breve romanza sino a quel momento poco considerata dalla critica, si è ormai in grado di ricostruire, per linee generali, le modalità con cui il bagaglio novellistico orientale sia arrivato in Occidente. Alla base del viaggio dei testi vi è, infatti, una biblioteca che accomuna Oriente a Occidente costituita da alcune opere capitali individuate da Angelo Michele Piemontese' ${ }^{1}$ :

- Il libro di Sindbad;

- Il romanzo greco di Alessandro;

- Il Panchatantra;

- Le 1000 Novelle;

- Barlaam e Josafat.

Posto che nel percorso verso Occidente si possono individuare varie "stazioni" nelle quali i testi originari subivano una sorta di rifacimento per essere adattati ai nuovi ricettori, rimane indiscusso che nel passaggio da Oriente a Occidente questi libri conoscono un vettore linguistico fondamentale quali l'arabo e l'ebraico per essere queste lingue confinanti con ogni area ${ }^{2}$. E se tali lingue sono dei buoni vettori, sembra che una delle stazioni privilegiate per il viaggio verso Occidente sia proprio la Castiglia medievale, insieme alla Sicilia. Si tratta di due stazioni decisive per la diffusione del testo in Occidente poiché proprio da qui partiranno versioni in lingua latina che permetteranno al Calila di viaggiare in tutto il mondo romanzo ${ }^{3}$. È, infatti, il Directorium humanae vitae a garantire il viaggio del testo in seno alla comunità romanza che ormai, all'altezza cronologica dei secoli XIII e XIV, era linguisticamente

\footnotetext{
${ }^{1}$ Angelo Michele Piemontese, «Narrativa medioevale persiana e percorsi librari internazionali», en Medioevo romanzo e orientale. Il viaggio dei testi. III Colloquio Internazionale (Venezia, 10-13 ottobre 1996), a cura di A. Pioletti e F. Rizzo Nervo, Soveria Mannelli, Rubbettino Editore, 1999, pp. 1-17, pp. 2-5.

${ }^{2}$ Così, infatti, osserva Piemontese, ivi, p. 11: «La traduzione libraria fra lingue di aree limitrofe, in determinate epoche, quali la sanscrita, la persiana, la siriaca e la greca, avveniva in linea diretta, e in quella indiretta per le aree via via contigue o lontane. Ma stazioni quali l'ebraica e l'araba, dislocate sulle tre fasce continentali, compresa la nordafricana, confinavano con ogni area. Prima dell'età rinascimentale, la trasmissione di testi persiani verso i paesi d'Europa transitava per l'area di dominio arabo, quasi un valico fisso da superare».

${ }^{3}$ Sui testi e sulle modalità di traduzione cfr. Elisabetta Paltrinieri, Il 'Libro degli Inganni' tra Oriente e Occidente. Traduzioni, tradizione e modelli nella Spagna alfonsina, Firenze, Casa Editrice Le Lettere, 1992, pp. 30-36.
} 
ben diversificata, ma unita solo dalla lingua latina, unico mezzo espressivo in grado di determinare contatti fra le diverse aree linguistiche romanze. Sono cinque i manoscritti oggi reperiti del Directorium, e numerosi gli esemplari a stampa, considerato che la prima edizione risale tra il 1484 e il 1493 (Strasburgo) dopo la quale altre ne sono seguite. Ciò indica il grande interesse del pubblico europeo (romanzo e non) verso tale testo anche ben oltre il Medioevo, ma è evidente che ciò sancisce la storia della diffusione del testo in quanto "libro"; non rende conto, cioè, della diffusione dei suoi racconti singolarmente, sganciati cioè dalla cornice. È proprio tale aspetto, invece, come già annunciato nell'introduzione, che s'intende qui verificare.

\section{La ricezione dei racconti. Alcuni dati}

Prima di procedere ad illustrare i risultati dei rilevamenti effettuati, con il relativo commento, è bene precisare che il corpus di riferimento si limita alla novella medievale. In altre parole, si prenderanno in considerazione solo le raccolte di novelle che non superano, come data limite, il secolo Xv. Dopo quella data la narrativa breve conosce una storia particolare in seguito alle mutate modalità di trasmissione dei testi che in questa sede non saranno presi in considerazione.

Per quanto concerne l'Italia, sei sono i grandi libri di novelle che costituiscono oggetto d'indagine per questo rilevamento: il Novellino duecentesco, il Decameron di Giovanni Boccaccio, il Trecentonovelle di Franco Sacchetti, il Paradiso degli Alberti di Giovanni Gherardi da Prato, le Porrettane di Giovanni Sabbadino degli Arienti, e il Novellino di Masuccio Salernitano. Il corpus novellistico italiano considerato conta di circa 620 novelle (dalle 9 novelle alle 300 del Sacchetti) ed è sorprendente notare come microtesti provenienti dal Calila e Dimna, o che presentino un tema simile ai racconti della raccolta orientale, siano davvero pochissimi; si è sull'ordine di 10 unità, pari al solo $2 \%$ del totale, distribuiti secondo il grafico seguente:

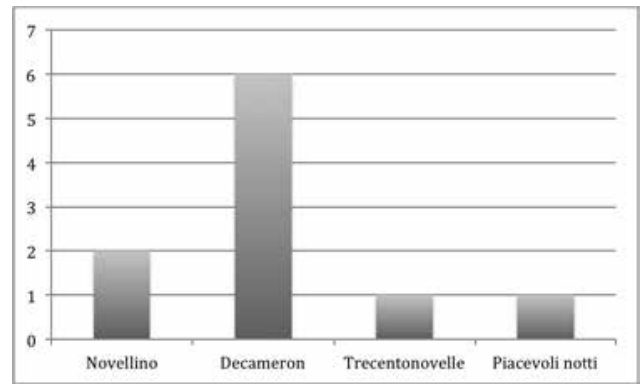


Se si escludono i libri di novelle medievali italiani che non presentano assolutamente novelle provenienti dal Calila e Dimna, il dato rimane sostanzialmente lo stesso: su un totale di 578 novelle, solo il 2\% sembra avere quale fonte la raccolta qui oggetto d'interesse. Si potrebbe concludere che la raccolta di origine indiana sia passata silente tra i lettori italiani, quand'anche essa sia stata tradotta in latino da Giovanni di Capua verso la fine del secolo XIII. Conforta sapere, rispetto a tali dati, che se non altro il testo indiano abbia avuto certa fortuna nella sua forma libro, mentre i singoli racconti difficilmente sono stati accolti quali motori per sviluppare storie presso i novellieri italiani medievali.

Nonostante ciò, merita senz'altro verificare quali racconti siano usciti dalla forma libro per ispirare i narratori italiani, e di esaminare da vicino quali siano stati gli ambiti tematici che hanno agevolato la ricezione dei singoli testi.

\section{I racconti, i temi e motivi}

Bisogna precisare che l'attribuzione della fonte a ciascuna rielaborazione italiana non va mai considerata certa; da questo punto di vista gli editori moderni sono piuttosto prudenti. Non è un caso, per esempio, che Alberto Conte parli di «riscontro» per la novella IX/12 e LXXIII del Novellino duecente$\mathrm{sco}^{4}$; Vittore Branca, a proposito del Decameron, riferisce di «vago riscontro» per la novella 2 della giornata II e di «nessun vero antecedente» per V 9 e VI $10^{5}$. Restare nel vago è un obbligo poiché nella trasmissione del Calila in Occidente non si è certi di ciò che è andato perduto, né è dato sapere a partire da quale momento il testo originario ha subito un processo di riscrittura più profondo. Siffatte prudenze sono inoltre necessarie poiché singoli racconti, dotati di vita propria e quindi sganciati dalla forma libro cui appartenevano originariamente, possono anche essere finiti in altre raccolte, sebbene questi siano di matrice orientale. Si pensi, per esemplificare, alla novella 4 della giornata VII, la novella di Tofano. La fonte più vicina a Boccaccio è senza dubbio l'esempio XIV della Disciplina clericalis ma lo stesso racconto è presente nel Directorium humanae vitae di Giovanni di Capua e nella versione latina del Kalila e Dimna di Raimondo di Béziers ${ }^{6}$.

${ }^{4}$ Il novellino, a cura di Alberto Conte. Prefazione di Cesare Segre, Roma, Salerno Editrice, 2001, p. 311 e p. 366 .

${ }^{5}$ Giovanni Boccaccio, Decameron, a cura di Vittore Branca, Torino, Einaudi, 1987, p. 141, n. 1, p. 681, n. 2 , p. 759 , n. 1 .

${ }^{6}$ In merito alla diffusione di questo racconto in Spagna si rimanda a María Jesús Lacarra, «Dalla donna ingannatrice alla malmaritata ingegnosa. Il racconto de Il pozzo (Decameron VII 4) alla luce della tradizione», en Id., Saggi sulla narrativa breve castigliana medievale, a cura di Gaetano Lalomia, Vero- 
Come si vede, simili problemi rendono ancora più complessa l'individuazione di un rapporto certo tra il Calila e le raccolte di novelle italiane prodotte nel Medioevo; in siffatta condizione il numero rilevato precedentemente si riduce ulteriormente a qualche esempio e nulla di più anche perché la rielaborazione del racconto originario viene trasformato in modo così radicale da rendere estremamente opaca la matrice. L'opacizzazione, in taluni casi, è complicata dalla capacità degli autori di unire più elementi tematici provenienti da testi diversi in una sola novella; si prenda il caso della novella 2 della giornata III del Decameron, quella di Agilulf, in cui la prima parte, quella dedicata all'astuzia del palafrenire, potrebbe trovare riscontro, a detta di Vittore Branca, nella favole del pittore e della moglie del mercante che dalla raccolta orientale del Kalila e Dimna è passata al Directorium. Per la seconda sezione, invece, la fonte, sempre a detta di Branca, potrebbe essere un racconto presente nel Dolopathos, e quindi si passa alla tradizione del Sendebar?

Fatte queste debite considerazioni l'unico aspetto che può essere messo in luce è il viaggio dei temi; se si danno per buone le considerazioni fatte dagli editori moderni, che in qualche modo hanno cercato di mettere in relazione il singolo racconto delle raccolte italiane con le potenziali fonti, quali possono essere stati i motivi che gli autori medievali italiani hanno potuto ripescare nel serbatoio del Calila? Un rapido sondaggio, illustrato nella seguente tabella, offre uno spaccato in tal senso:

\begin{tabular}{|ll|}
\hline Novellino IX/”2 & Furbizia \\
Novellino LXXIII & Furbizia \\
\hline Decameron II 2 & Rovescio di fortuna \\
Decameron III 2 & Rovescio di fortuna \\
Decameron V 9 & Bon gesto premiato; rovescio di fortuna \\
Decameron VI 10 & Astuzia \\
Decameron VII 4 & Furbizia delle donne \\
Decameron VII 8 & Furbizia delle donne \\
\hline Trecentonovelle novella CXXXV & Rovescio di fortuna \\
\hline
\end{tabular}

L'area tematica prevalente è senz'altro la "furbizia", ovvero quei casi in cui i protagonisti, ricorrendo alla propria intelligenza, riescono a sopravvivere a situazioni a loro sfavorevoli. Si tratta di un tema che è direttamente collegato anche all'altro ambito tematico ricorrente, il "rovescio di fortuna".

na, Edizioni Fiorini, 2010, pp. 51-78.

${ }^{7}$ Giovanni Boccaccio, Decameron, ed. cit., p. 388, n. 2. 
In entrambi i casi, i protagonisti riescono, per sorte o per propria capacità, a mutare la condizione di partenza del tutto sfavorevole. Un minimo posto viene riservato alla "furbizia delle donne", un tema piuttosto ricorrente nei testi letterari medievali (italiani e non), che tuttavia la novellistica italiana non pare attingere dal Calila e Dimna, bensì da altre fonti.

Come si vede, si tratta pur sempre di temi che generano un forte contatto tra $\mathrm{i}$ testi, e che in certa misura spiegano e giustificano le ragioni della ricezione del testo orientale in Occidente. Alla base del viaggio del Panchatantra verso Occidente, infatti, risiede una facilità di adattamento dei contenuti in stazioni e in epoche diverse. L'Occidente romanzo non è insensibile al tema/ motivo della furbizia delle donne, così come non è estraneo a entrambi gli universi culturali la furbizia in quanto motore narrativo. È quindi il motivo a generare l'accoglienza del testo, e in questo specifico caso gli scrittori italiani si sono lasciati sedurre da quegli aspetti che meglio si sarebbero potuti piegare alla verve narrativa. Si prenda il caso, citato anche oltre, della novella 2 della giornata III, quella del re Agiluf. Giovanni di Capua aderisce pienamente allo scheletro narrativo del Kalila wa Dimna, mutando solo il mestiere del marito della moglie: da mercante diviene falegname. Per il resto, non solo si mantiene la stessa struttura, ma finanche lo stesso fine con cui viene raccontata la storia, cioè dimostrare come sia opportuno non agire affrettatamente.

La novella di Boccaccio, non essendo una "traduzione", propone maggiori cambiamenti, in taluni casi anche assai vistosi, come il ruolo sociale del protagonista maschile che da falegname (supponendo che il Directorium sia la fonte alla quale Giovanni Boccaccio abbia attinto) diviene un monarca. Siffatto cambiamento non appare del tutto casuale poiché Boccaccio giostra la narrazione tutta sulla diversa scala sociale tra Agilulf e il suo rivale. È proprio in tale aspetto che va colto, a mio avviso, la metamorfosi dello scheletro narrativo, nel voler fare della furbizia una sorta di valore positivo poiché il «pallafreniere» non solo riesce a giacere con la moglie del re, ma riesce, finanche, a sottrarsi alla vendetta del re. E che la novella sia tutta concentrata sul motivo della furbizia lo dimostrano le particolari attenzioni rivolte alle azioni dello stalliere quando si prepara per incontrarsi con la regina, conformemente al tema della terza giornata nel corso della quale si narra «di chi alcuna cosa molto da lui disiderata con industria acquistasse o la perduta ricoverasse» (III 2, 29).

Ma la novella di Boccaccio pare recuperare molto di più dalla fonte; a una prima lettura il motivo della furbizia pare essere adattato, nelle parole della regina della giornata sopra citate, alla celebrazione di chi riesce a ottenere ciò che si desidera, ma in realtà il finale del racconto recupera la ragione 
prima del racconto del falegname, della moglie e del pittore, cioè non agire frettolosamente. Avendo scoperto Agilulf di essere stato tradito, decide di incriminare il palafreniere attraverso una prova certa, e così gli rade la testa. Costui, rendendosi conto che in tal modo sarebbe stato scoperto, contrattacca rasando la testa a tutti gli altri stallieri che dividevano la stanza con lui. Agilulf, non solo viene beffato, ma è costretto al silenzio altrimenti avrebbe dovuto rivelare la ragione per cui tutti i palafrenieri sarebbero stati accusati, come lo stesso Boccaccio dice in chiusura della novella:

Un altro gli avrebbe voluti far collare, martoriare, essminare e domandare; e ciò facendo avrebbe scoperto quello che ciascun dee andar cercando di ricoprire, e essendosi scoperto, ancora che intera vendetta n'avesse presa, non iscemata ma molto cresciuta n'avrebbe la sua vergogna e contaminata l'onestà della donna sua (III 2,31).

Detto diversamente, e riprendendo la ragione per la quale Dimna narra questa storia, se il re avesse agito d'istinto, dominato dalla rabbia, il peccato sarebbe ricaduto su di lui.

\section{Il Calila e Dimna non ha insegnato a narrare}

Da questo quadro definitivo emergerebbe che in Italia Calila e Dimna, parafrasando un contributo di María del Pilar Palomo ${ }^{8}$, non ha insegnato a narrare. Non solo, emergerebbe che la raccolta indiana sia passata in totale silenzio e che abbia lasciato pallide tracce nella novellistica peninsulare. Siffatte conclusioni sarebbero piuttosto semplicistiche e forvianti.

In prima battuta bisogna rilevare che al pari di altre collezioni di racconti orientali, quali ad esempio la Disciplina clericalis o il Sendebar, il Calila e Dimna ha avuto più successo in quanto opera unitaria che non in quanto collettore di racconti ${ }^{9}$. Se si pensa alla sua diffusione nel Medioevo romanzo si comprende bene quanto si viene affermando: giunto nella versione araba di Ibn al 'Muqaffa, tradotto in latino da Giovanni di Capua con il titolo $D i$ rectorium humanae vitae - tradotto da una versione ebraica, almeno stando a

\footnotetext{
${ }^{8}$ María del Pilar Palomo, La novela cortesana (Forma y estructura), Barcelona, Editorial Planeta, 1976, p. 33.

${ }^{9}$ In merito alla diffusione dei racconti della Disciplina clericalis in Italia mi permetto di rinviare a Gaetano Lalomia, «Dalla Disciplina clericalis all'Italia. Viaggio di testi, viaggi di cultura e identità culturale», in Culture, livelli di cultura e ambienti nel Medioevo occidentale. Atti del IX Convegno della Società Italiana di Filologia Romanza (Bologna, 5-8 ottobre 2009), a cura di Francesco Benozzo, Giuseppina Brunetti, Patrizia Caraffi, Andrea Fassò, Luciano Formisano, Gabriele Giannini, Mario Mancini, Roma, Aracne Editrice, 2012, pp. 579-602.
} 
quanto recita il prologo ${ }^{10}$, il testo ha iniziato a circolare immediatamente per tutta l'Europa. È questa versione che inevitabilmente permette il passaggio del testo nella penisola italiana, lasciando comunque delle tracce indelebili nei novellieri italiani medievali. Basti un esempio su tutti: la novella 2 della III giornata. Ciò che lascia intravedere la dipendenza della novella decameroniana dal racconto del Directorium è proprio ciò che esclama la regina quando ritiene che il marito ritorni per giacere nuovamente con lei dopo aver già consumato il rapporto sessuale:

Directorium humanae vitae (cap. III)

Quid tibi nocte ista et quare rediisti ad me iterum festinanter, ex quo adimplevisti tuam voluntatem? At ille cum audisset hoc verbum, percepit, quomodo alius homo accessit ad eam nocte illa
Decameron, III 2 (17-18, p. 342)

«O signor mio, questa che novità è stanotte? Voi vi partite pur testé da me e oltre l'usato modo di me avete preso piacere, e così tosto da capo ritornate? Guardate ciò che voi fate». Il re, udendo queste parole, subitamente presunse la reina da militudine di costume e di persona essere stata ingannata $[\ldots]$

Come si può notare da un puntuale riscontro, non vi sono dubbi sul fatto che Boccaccio abbia dinanzi tale testo: l'accenno alla notte, il re che presto se ne va dalla stanza della regina, il rendere l'atto sessuale con una metafora per cui il «voluntatem» del testo fonte si tramuta in «piacere», mi paiono indizi sicuri del debito contratto dall'autore del Decameron.

Non si tratta solo di tale passaggio, ma Boccaccio ha altresì mantenuto vivo lo scontro tra $i$ due contendenti, impegnati rispettivamente nel loro obiettivo erotico e a difendere la propria posizione legittima di sovrano nel caso di Agiluf.

Nonostante tale puntuale riscontro, rimane comunque da verificare come mai le storie di Calila e di Dimna non siano riuscite a penetrare del tutto nella penisola italiana se sganciate dalla forma libro che le contiene. Forse ha inciso proprio il passaggio della traduzione proposta da Giovanni di Capua che,

\footnotetext{
${ }^{10}$ «Cum plura diversarum scientiarum genera esse prospexerim in lingua fundata hebraica non parvae utilitatis in eruditionem christianorum consortii, ut in sacris scripturis et divinis, moralibus atque medicinalibus, ipsa ex praedicta lingua in latinam reducere meus animus adspiravit. Inter quae nunc hunc libellum dictum KELILA ex illa lingua in hanc, nunc esse vidi non etiam immerito transferendum; est enim opus virorum intelligentiae animarum multae informationis et nihil earundem non modicae delectationis» (Iohannes de Capua, Directorium humanae vitae alias parabolae antiquorum sapientum, ed. di V. Puntoni, Pisa, Nistria, 1884, Prologus. Si cita dalla versione on line http://www.hs-augsburg. de/ Harsch/Chronologia/Lspost13/IohannesCapua/cap_dipl.html).
} 
in realtà, si tinge di toni particolari che le altre versioni sembrano non avere; si veda il prologo:

Ad honorem autem divinae Trinitatis sanctissimique eius nominis exaltationem, salutem et meritum animae, fortitudinem corporis et roborationem, atque dierum productionem reverendi patris et domini, domini Mathei Dei et apostolicae sedis gratia tituli sanctae Mariae in porticu diaconi cardinalis, motus sum praesens opusculum in lingua latina interpretari ${ }^{11}$.

Aver posto il lavoro di traduzione sotto il tutorato del cardinale Matteo di Rubeis implica aver impresso all'opera un valore altamente morale e averla inserita in un orizzonte d'attesa che era in grado di fruire il valore etico che il testo prospettava. Gli insegnamenti divulgati nell'opera seguono un ordine che evidentemente imprime ai microtesti un valore che difficilmente poteva subire un'interpretazione e una lettura diverso. Altro è il tono del Calila castigliano duecentesco:

Los filósofos entendidos de qualquier ley et de qualquier lengua siempre punaron et se trabajaron de buscar el saber, et de representar et hordenar la filosofía, et eran tenudos de fazer esto. [...] Et posieron [i filosofi] enxenplos et semejanças en la arte que alcançaron et llegaron por alongamiento de nuestras vidas et por largos pensamientos et por largo estudio ${ }^{12}$.

Per quanto il valore morale sia pur messo in evidenza, il prologo di questa versione orienta il lettore verso il "sapere" quale strumento fondamentale non solo della conoscenza, bensì del "reggimento" del proprio vivere. Come si vede, i due testi sono proposti al pubblico con intenti ben diversi; mentre infatti la versione castigliana mantiene vivo il messaggio socio-politico originario del testo, derivato con molta probabilità dalla versione araba dalla quale si traduce, Giovanni di Capua pare sminuire e assottigliare tale senso per riorientare il testo verso ben altra direzione (orientamento che con molta probabilità era già stato impresso nella versione in lingua ebraica dalla quale di Capua traduce). Non v'è dubbio che il veicolo morale del testo, così come l'ha impresso Giovanni di Capua, è rimasto pressoché intatto nel tempo, sebbene tanto Agnolo Firenzuola quanto Anton Francesco Doni, nel secolo XVI,

\footnotetext{
${ }^{11}$ Ivi.

${ }^{12}$ Calila e Dimna, ed. di Juan Manuel Cacho Blecua y María Jesús Lacarra, Madrid, Castalia, 1984, p. 89. Si precisa che tale prologo è trasmesso solo dai manoscritti B e P.
} 
imprimano alla raccolta di novelle un senso diverso da quello originale ${ }^{13}$. Così, infatti, sollecita Firenzuola le «cortesi donne»:

Pigliateli adunque con lieta fronte; e quando l'ago e 'l fuso faran con voi triegua, leggeteli come per via di diporto $[\ldots]^{14}$.

Le storie di Calila e di Dimna, pertanto, hanno un destinatario privilegiato, le donne, e servono per intrattenere il lavoro domestico, là dove, invece, Anton Francesco Doni indirizza le stesse storie a ben altro fine («perciò che le istorie, novelle e favole son molto utili e maestrevoli $\rangle^{15}$ ). Uno, riprendendo l'idea di novella che intrattiene le donne mutuandola da Boccaccio, seleziona il pubblico dando ai racconti il valore d'intrattenimento, l'altro, invece, recependo il fondo morale dei racconti di Calila e di Dimna, ne sottolinea l'utilità didattica. In pratica, le riscritture cinquecentesche del Calila e Dimna dialogano inevitabilmente con il modello decameroniano e, soprattutto, con quella zona del Decameron che genera un confine tra raccontare, dialogare, conversare e commentare, tutti elementi presenti nel Calila e, come ha rilevato Renzo Bragantini, fondanti per il testo boccacciano ${ }^{16}$. Ecco che allora Calila e Dimna hanno insegnato a perimetrare i momenti tra dialogo, racconto, conversazione e commento, dando così alla novellistica medievale italiana la misura del novellare a cornice.

A poter aver reso meno permeabile i microtesti nella novellistica italiana medievale può anche aver contribuito lo statuto della novella in quanto tipo di testo. La novella italiana, nel corso del Tre e del Quattrocento, assume sempre più il carattere di racconto d'intrattenimento che si concentra su due aspetti fondamentali: la battuta arguta, e personaggi che si muovono in un ambiente familiare, assai prossimo al lettore. I tratti peculiari assunti dalla novella rendono così il serbatoio tradizionale orientale meno fruibile di per sé, a patto di essere del tutto assimilato al genere novella. È, pertanto, una questione di poetica; la novella, in quanto forma letteraria, infatti, si codifica nel Medioevo italiano a partire dai vari tipi dei genera narrationum, per

\footnotetext{
${ }^{13}$ In merito alla ricezione del Calila e Dimna da parte dei novellieri italiani si rimanda a María Jesús Lacarra, «La fortuna del Exempario contra los engaños del mundo en Italia», en España al revés. Atti del I Convegno di Studi Interdisciplinari (Ragusa Ibla, 4-5 aprile 2006), a cura di Anita Fabiani, Quaderni del Dipartimento di Filologia Moderna, Catania, 2008, pp. 23-43.

${ }^{14}$ Agnolo Firenzuola, La prima veste dei discorsi degli animali, in Id., Le novelle, a cura di Eugenio Ragni, Roma, Salerno Editrice, 1971, pp. 207-299, pp. 207-208.

${ }^{15}$ Anton Francesco Doni, La moral filosofia. Trattati, in Id., Le novelle, tomo I, a cura di Patrizia Pellizzari, Roma, Salerno Editrice, 2002, p. 9.

${ }^{16}$ Renzo Bragantini, Vie del racconto. Dal Decameron al Brancaleone, Napoli, Liguori Editore, 2000, pp. 17-18.
} 
assumere immediatamente tratti propri e ben codificati già tracciati nel prologo del Novellino duecentesco:

Voi ch'avete i cuori gentili e nobili infra li altri, parlando, onorando e temendo e laudando quel Signore nostro che n'amò prima che elli ne criasse, e prima che noi medesimi ce amassimo. E [se] in alcuna parte, non dispiacendo a lui, si può parlare, per rallegrare il corpo e sovenire e sostentare, facciasi con più onestade $\mathrm{e}$ [con] più cortesia che fare si puote. E acciò che li nobili e gentili sono nel parlare e ne l'opere quasi com'uno specchio appo i minori, acciò che il loro parlare è più gradito, però ch'esce di più dilicato stormento, facciamo qui memoria d'alquanti fiori di parlare, di belle cortesie e di belli risposi e di belle valentie, di belli donari, e di belli amori, secondo che per lo tempo passato hanno fatto già molti ${ }^{17}$.

È dato rinvenire due modi di parlare, uno serio, di tono elevato, e uno meno impegnato, atto a intrattenere di cui il Novellino dà mostra radunando, non a caso, «i fiori di parlari» con l'intento di mettere a frutto una retorica della parola alta per tematiche non altrettanto elevate. Come osserva Michelangelo Picone, si trattava di «stabilizzare e innalzare i gusti del pubblico, e al tempo stesso di garantire una dimensione autoriale» ai codificatori del genere novella ${ }^{18}$. Non è un caso che dal Novellino si passi direttamente a Giovanni Boccaccio, cioè a colui che ha dato assetto definitivo alla novella portandola al punto elevato in cui essa poi si viene a trovare. E non è un caso che proprio egli sia la voce autoriale che maggiore uso fa delle forme narrative brevi allora vigenti per stravolgerne lo statuto e dando loro un assetto formale novellistico. È proprio della poetica della novella recuperare storie che trattano della vita quotidiana, di puntare sui fatti, per cui anche quando si recuperano temi e situazioni dai testi orientali, questi vanno immediatamente resi fruibili stravolgendone l'ambientazione per renderli più familiari ai destinatari del racconto $^{19}$.

Sulla base di tali osservazioni pare del tutto normale considerare che Kalila e Dimna più che non aver insegnato a narrare, hanno fornito modelli di narrazioni, forme del narrare, che veri e propri testi. I racconti dei due sciacalli rientrano in quelle scelte possibili che si offrono all'autore di un testo, ma evidentemente non sono facilmente asportabili in quanto microtesti

\footnotetext{
${ }^{17}$ Cito da Il novellino, ed. cit.

${ }^{18}$ Michelangelo Picone, Boccaccio e la codificazione della novella. Letture del Decameron, Ravenna, Longo Editore, 2008, p.15.

${ }^{19}$ Enrico Malato, «La nascita della novella italiana», in La novella italiana. Atti del Convegno di Caprarola (19-24 settembre 1988), 2 voll., Roma, Salerno Editrice, 1989, vol. 1, pp. 3-45, p. 25.
} 
da riusare, o lo sono molto poco. In un certo senso, tanto il Calila e Dimna quanto la Disciplina clericalis si presentano ai lettori italiani con un impianto fortemente didattico che se poteva funzionare bene a livello di ricezione completa del testo, non pare sia però utile per trarre spunto per la riformulazione di novelle. Non è un caso, mi pare, che della Disciplina clericalis la novellistica italiana abbia riutilizzato i microtesti più narrativi, quelli cioè più assimilabili dalla poetica della novella ${ }^{20}$.

Recibido: 26/03/2015

Aceptado: 11/06/2015

${ }^{20}$ Gaetano Lalomia, «Dalla Disciplina clericalis all'Italia...», cit. 
$\cos$

La Ricezione Dei Racconti del CaLILA E Dimna In Italia

Resumen: El artículo analiza la recepción de algunos cuentos del Calila e Dimna en la novella italiana de la Edad Media, con el fin de detectar los cuentos que pasan a la novella. Según lo que el análisis de los textos sugiere, estos no son muchos y los pocos que se utilizan sufren cambios considerables; se intenta averiguar las razones que han determinado el aparente escaso interés hacia los cuentos del Calila e Dimna por parte de los novellieri italianos.

Palabras Clave: Cuentos. Calila e Dimna. Novella italiana.

\section{The Reception of CALILA E DimNa FABles in Italy}

ABSTRACT: The article analyzes the reception of some Calila e Dimna short stories in the Middle Ages Italian novella; the intent is to find the short stories that passed to the Italian novella. According to the results of this investigation, few are the fables that are used by Italian authors and when they use some of them they deeply change them; in the article I try to understand the reasons why Calila and Dimna appears less interesting as a collection of fables than as book, according to the Italian novellieri.

KeYwords: Short stories. Calila and Dimna. Italian novella. 